\title{
“Hands-on" Remote Sensing of Physical Models in Exploration of Surficial Processes ${ }^{1}$
}

\author{
Jeffrey J. Clark \\ Department of Geology \\ Lawrence University
}

\begin{abstract}
The goal of this work is to create an interactive, data-rich learning environment that will enhance student understanding of remote sensing principles and surficial processes. In service of this goal a data acquisition system consisting of common consumer electronics (e.g. a digital camera and Microsoft Kinect) was used to monitor temporal and spatial changes in a scale model of a fluvial setting. Analysis of these data allows students to track landform evolution in response to changing inputs and boundary conditions. These scale-model observations of surficial processes can be also compared to full-scale planetary landscapes.
\end{abstract}

\section{Introduction}

A common way of engaging students with the sciences in the laboratory setting is through experimentation. But experimentation is challenging in the earth sciences because the temporal and spatial scales involved make direct observation of many geological processes problematic. Physical models, however, allow us to greatly compress the temporal and spatial scales, make direct observations, visualize processes, and control the inputs and boundary conditions allowing us to create "what if" scenarios. The advent of inexpensive data acquisition instruments such as digital cameras and the Microsoft Kinect ${ }^{\mathrm{TM}}$ allow us to easily and inexpensively take frequent detailed measurements of these models. Moreover, the familiarity of these everyday consumer electronic devices provides a bridge to discussion of applications of the more sophisticated space-based remote sensing assets monitoring Earth and other planets.

Remotely sensed data is ubiquitous in the technologically-based world we live. We encounter remotely sensed data on the news in the form of satellite imagery of clouds and water vapor, we use digital maps and imagery our smart phones to find restaurants, and we can now view the entire surface of Mars using Google Earth ${ }^{\mathrm{TM}}$. However, few people realize where these data come from and how it is processed for our use. Another goal of this work therefore is to familiarize students with the principles of remote sensing by having them collect and analyze their own data.

The laboratory activities based on the use of remote sensing of physical models and of planetary surfaces described below provide a dynamic and exciting learning environment, which is vital for engaging the student scientist and developing scientific habits of mind. Data-rich environments like those developed here establish a context for exploration, and require students to quantify observations, propose explanations, test ideas, and communicate their results (AAAS, 1989; NRC, 1997). The open-ended research questions enabled through investigation of physical models also hone critical thinking skills and motivate students to take ownership of the material

\footnotetext{
${ }^{1}$ Funding provided by NASA's Higher Education Program and distributed through the Wisconsin Space Grant Consortium. Additional funding provided by the Lawrence University Excellence in Science Fund.
} 
(AGU, 1997). At its best, participation in discovery-based laboratory exercises enhances the students' ability to relate to, analyze, and understand real world data and processes they are investigating (Boyer Commission, 1998). These student experiences are essential as we train the next generation of scientists and a technologically literate work force.

Work on this project began in the summer of 2013 and continued throughout the 2013-14 academic year. The modeling environment and data acquisition system was developed over the summer and laboratory activities and quantitative exercises were developed for two courses offered during the academic year. The sections below describe these activities in detail.

\section{Modeling Environment and 2013 Summer Work (4 students)}

We used a 2.5 meter-long, 1.2 meter-wide and 0.20 meter-deep soapstone table as the base of the flume. The table can be tilted to slopes of $\sim 5 \%$ (Figure 1). Water is supplied to a constant head

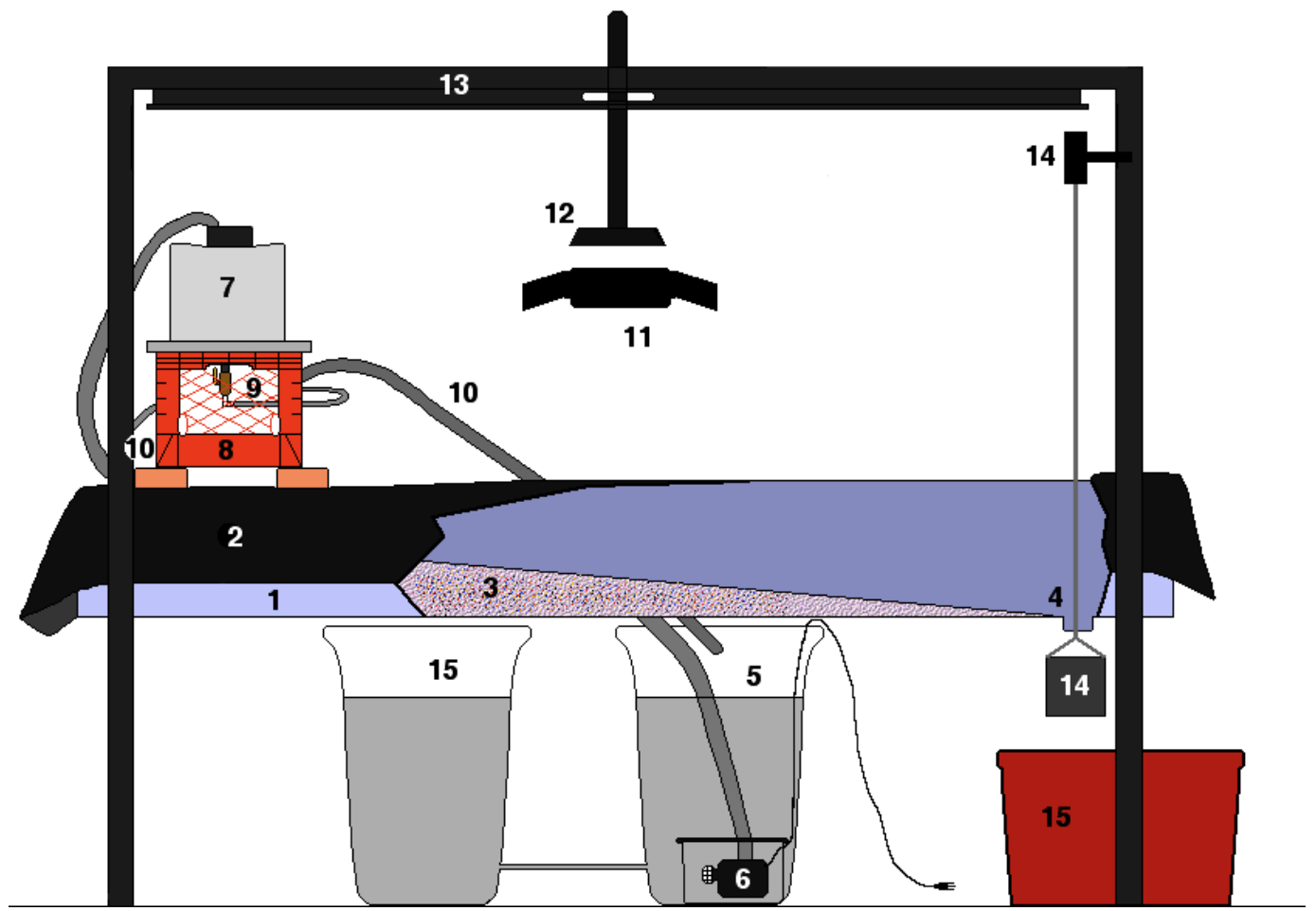

Figure 1 - Schematic diagram of flume components.

1 Flume base - soapstone.

2 Impermeable liner over flume base

3 Ground plastic "sediment" on top of liner

4 Drain and weir

5 Reservoir: holds water to be circulated through the system

$6550 \mathrm{gph}$ pump inside filter box

7 Constant Head Tank

8 Constant Head Tank Stand ( $2 \times 4$ s and milk crate)

9 Valve at base of head tank to control discharge to flume.

10 Discharge Tube, Dispersion Mechanism, and Overflow Tube

11 Lighting: 2X3000W-equivalent LED arrays

12 Kinect and DSLR

132 " circular steel pipe frame supports soapstone and used to mount instrumentation

14 Sediment Weigh Mechanism (Load cell, chain, and sieve)

15 Additional reservoirs 
tank via a 550gph pump. The water drains to the back to pump reservoir for recirculation. The sediment in the flume consists of ground thermoset plastic (Figure 2). This is a durable material that has two distinct advantages over quartz/feldspathic sands. The first is that it has a specific gravity of 1.55 . The lower density ( $\sim 58 \%$ of quartz) means that it will move more easily. The other advantage is that the plastic is color-coded by size, which means that we can use digital color photography and image processing to quickly determine the surface composition. The data acquisition system consists of a Microsoft Kinect ${ }^{\mathrm{TM}}$ which is used to take 3-D images and a 20MP digital color camera (DSLR) which is used for color photography and provides the images for textural analysis. An 8" diameter by 8 " deep circular sieve with a mesh size of $0.074 \mathrm{~mm}$ collects the sediment that passes out the drain. The sieve is suspended by a chain and connected to a data-logging load cell which allows us to track sediment evacuation from the system over time. The collected sediment can be mechanically sorted and the size distribution of bulk transport can be determined.

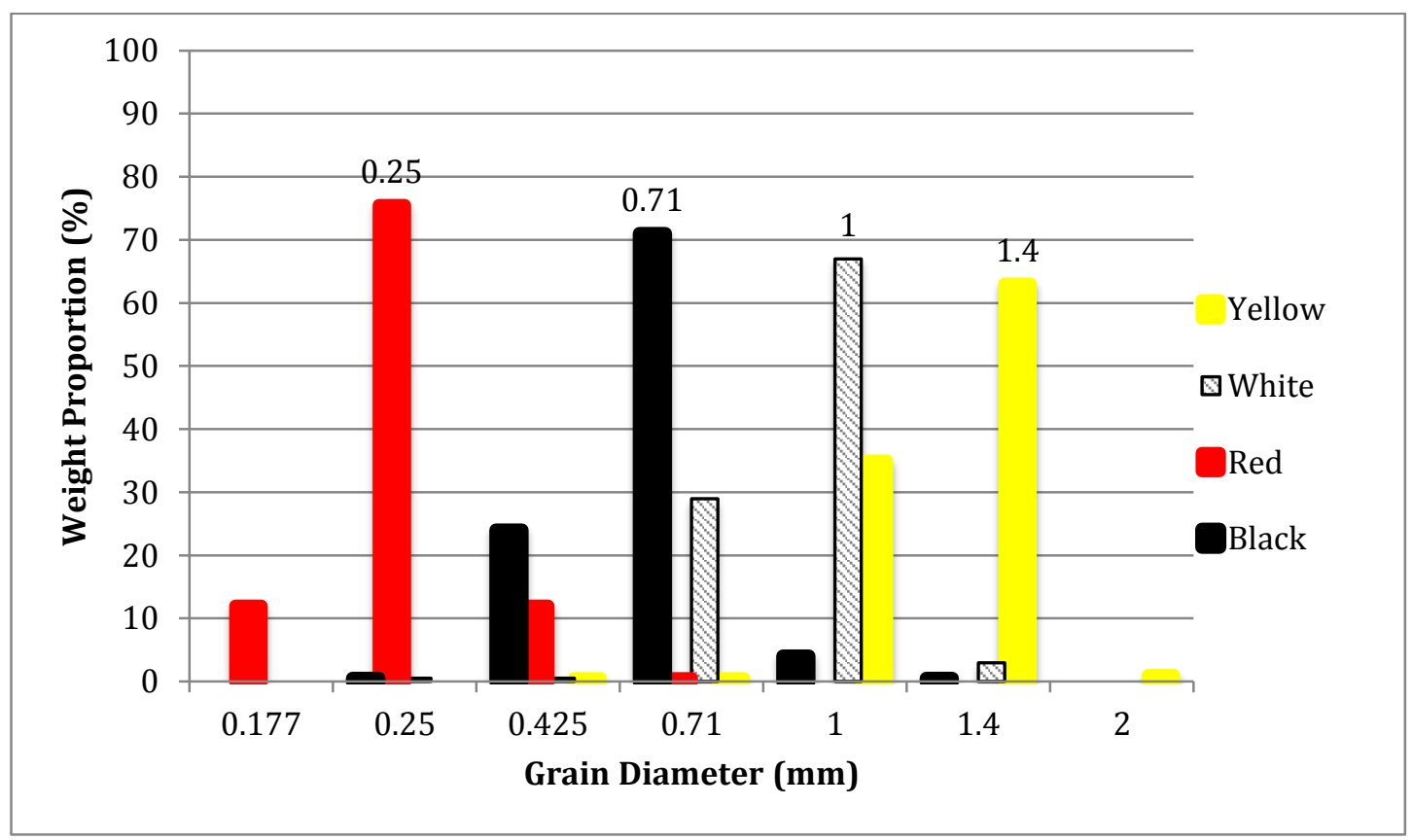

Figure 2. Sediment size distribution of colored sediment.

In the summer of 2013, a team of four students worked to develop a modeling environment which could be use to simulate fluvial processes. One student designed and built the constant head tank to control the input of water and calibrated the force gage to record sediment flux. Another was responsible for developing protocols to turn the color digital photography into texture maps based on sediment color. A custom data acquisition program was developed by another student to tap the Kinect's data stream and to set intervals to record and time stamp data using protocols outlined by Mankoff and Russo, 2012. The fourth student measured planform metrics of terrestrial and Martian alluvial meanders such as wavelength, width, and radius of curvature and developed curves for comparative purposes (Figure 3). At summers end these efforts were compiled into a user manual for the flume and a protocol was developed for efficient and inexpensive processing and analysis of the data. The following activities were developed. 


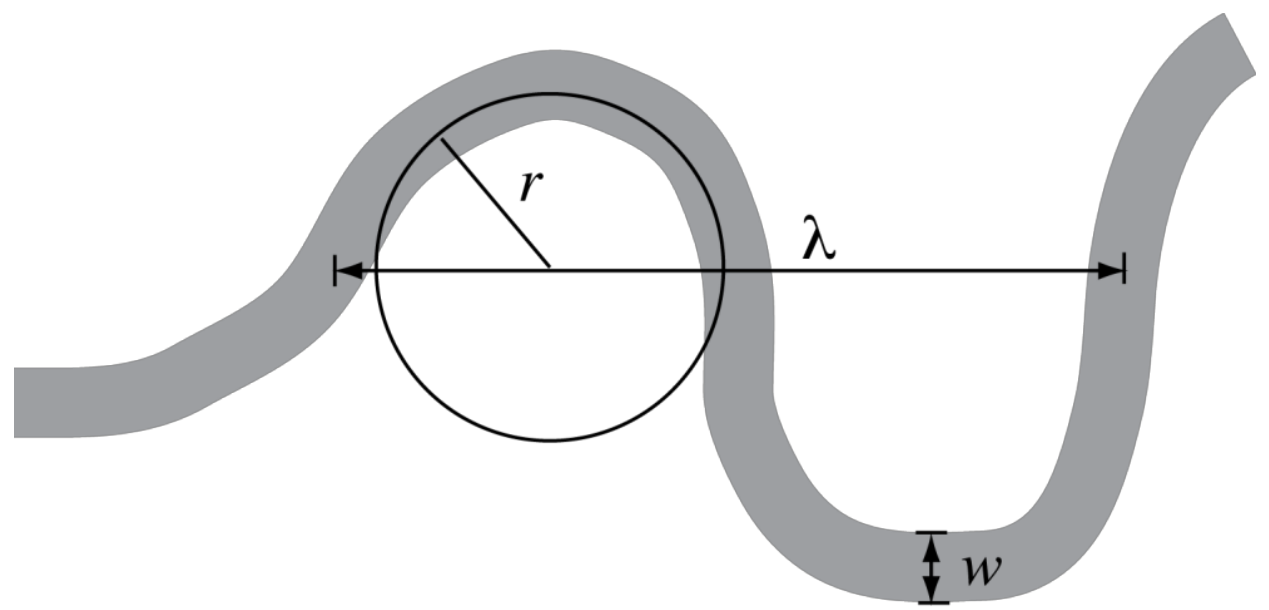

Figure 3. Schematic diagram of meander metrics measured. Where $w$ is the channel width at bankfull, $\lambda$ is the wavelength and $r$ is the radius of curvature. Image from Richeson.

\section{Course Activities}

Several activities were built around the concepts of using hands-on remote sensing techniques as an inroad to exploration of space-based remote sensing and on comparing surficial processes on Mars and Earth. The activities were incorporated into two courses as described in the following sections.

\section{Course 1: GEOL 360 - Earth Surface Processes (12 students)}

Two labs have been developed for this course related to this project. The first involves comparing meander characteristics (Figure 3) from aerial imagery of Earth and Mars. We used the measurement tools in ArcGIS (ESRI, 2011) for our work, but it could have easily been done in GoogleEarth or any location aware image processing program. The advantage of ArcGIS is that terrestrial imagery can be accessed easily via ArcGIS Online (www.arcgis.com/home/) and spatially referenced Martian imagery from the university of Arizona's HiView application (www.uahirise.org/hiview) can be downloaded. (The resolution of imagery in GoogleEarth - Mars is too coarse for analysis of smaller channels.) Previous work on terrestrial streams has shown strong correlations between meander wavelength $(\lambda)$ and channel width $(w)$, and meander wavelength and radius of curvature $(r)$ (Leopold and Wolman, 1960 and Williams, 1986). Martian channels also exhibit strongly correlated relationships between these metrics, though there are a few caveats to keep in mind. Martian meanders, for example, are relict features and true channel widths of alluvial channels on Mars are unknown because the floodplains have been eroded away by wind leaving the coarser channel beds in relief (Pain and Ollier, 1995). Nonetheless students can compare the terrestrial metrics and correlations with those of Martian channels and speculate on past Martian climate and environment.

In the second lab activity students simulate sediment transport dynamics and resultant landform evolution in response to changing input conditions. Stream systems will change in response to changes in base level, valley slope, or the supply of water and sediment to the reach. The changes in input variables, in turn are caused by larger drivers such as regional tectonics and climate change. The modeling system developed here is highly adaptable allowing us to simulate changes from any of these drivers. For example, increasing the weir height will simulate a rise in base level, which can be driven by subsidence, normal faulting, or eustatic adjustment due to climate 
warming. Lowering the weir height simulates base level lowering which is a product of isostatic uplift, thrust faulting, or a glacial climate phase. Basin wide tectonics and tilting can be modeled by changing the slope of the flume. Climate change or changes in land use can be simulated by changing the magnitude of the water or sediment discharge supplied to the flume. In each case the stream will adjust its size, shape, or planform in response.

The goal of the lab is for students to predict the changes that will take place in response to changing inputs, and then collect and analyze data to assess their predictions. We use the Kinect to track temporal changes in surface topography and the DSLR to quantify how surface sediment is sorted. The sieve and load cell allows us to track sediment leaving the system. By comparing the image sequences and the record of sediment transport out of the channel against the change in driving variables students are provided a data rich exercise with open-ended questions. Students report both qualitative and quantitative descriptions of system response and propose the mechanism of the response.

The Kinect yields Portable Grey Map files, which are basic Digital Elevation Models (DEMs) in a simple raster with the z-variable as distance. Once the images are calibrated and corrected, they can easily be analyzed in FIJI (Schindelin et al., 2012) or other image processing programs to show channel topography in 3-D (Figure 4). We use FIJI because of the wide variety of functions

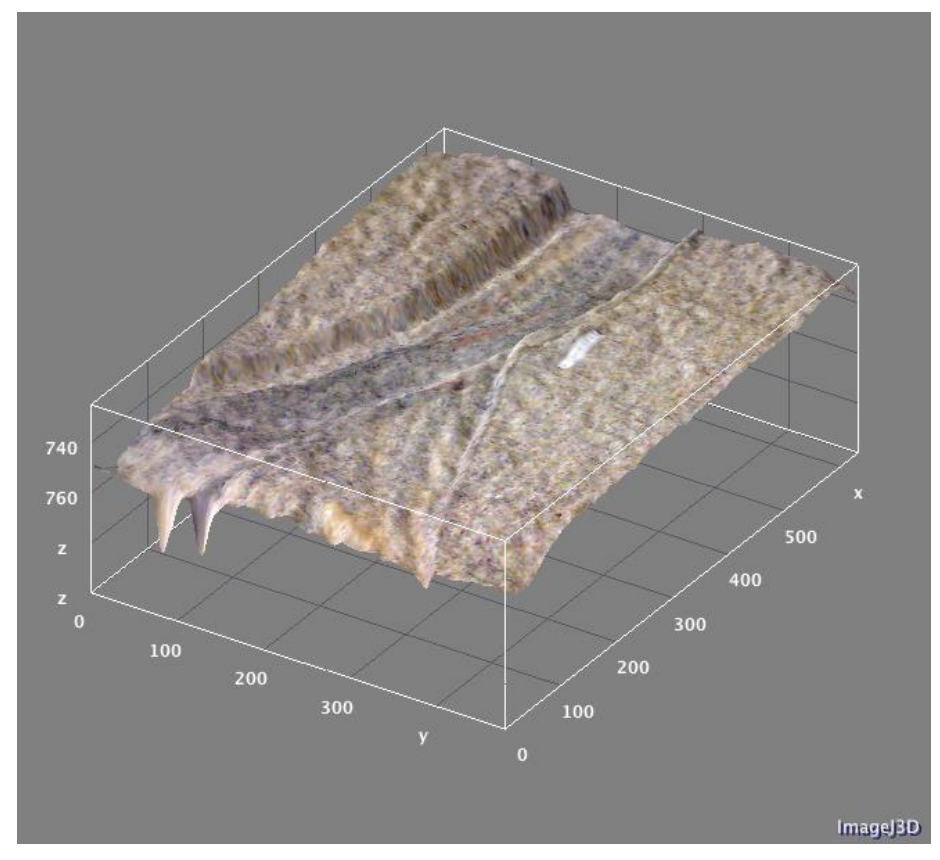
available, the broad user support base, and because it is freely available and works on a variety of operating systems (fiji.sc/Fiji). Because these are simple raster images, subtraction of one image from another quickly highlights areas of elevation change. Figure 5 shows an image where a later surface was subtracted from the initial conditions. Warmer colors indicate areas of erosion, which occur as the channel migrates the outside bank. Students can measure the distance migrated on the calibrated image and volumes of sediment eroded or deposited can be calculated and compared to those moved out of the system. These same patterns of stream migration are observed in a subsequent lab in the field and can be tied back to the

Figure 4 - Three dimensional data (pgm) from the Kinect with a digital photograph (ppm) overlaid. meander metrics lab described earlier as well. Time-lapse animations of the meander trace also help students see how migration occurs over time. Seeing these processes in real time helps the students visualize a process that in nature occurs sporadically and over decades. 

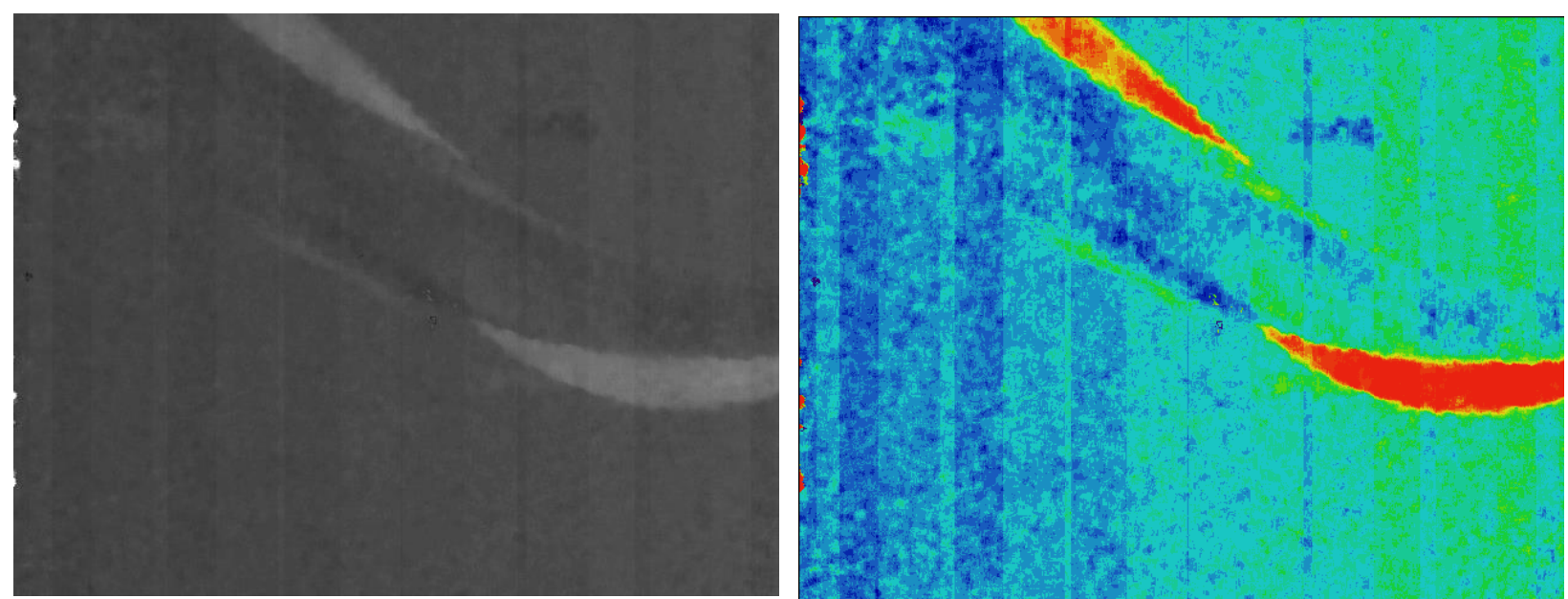

Figure 5 - Time differenced image showing areas of erosion at cutbanks shown as white regions in the grey scale map to the left and orange-red in the heat-map image to the right.

The color digital camera images are in a raster format called a Portable Pixel Map (ppm). We use the 20MP camera rather than the color image from the Kinect because the red and black grains are not visible in the $640 \mathrm{X} 480$ Kinect image. The images can be viewed and analyzed in FIJI and a FIJI plug in called "Weka Segmentation" (Hall and others, 2009) allows us to classify the image based on color (Figure 6). From these classified images we can determine the proportion of each color that makes up the surface of an area of interest. The ability to track surface textural changes over time combined with areas of erosion and deposition allows the students to see how erosion may leave behind coarse material and how material fines on the inner portion of the meander bends. These are observations they can also make in the field, but using the model allows them to see the sorting develop, whereas in the field we can only see the results of the sorting.
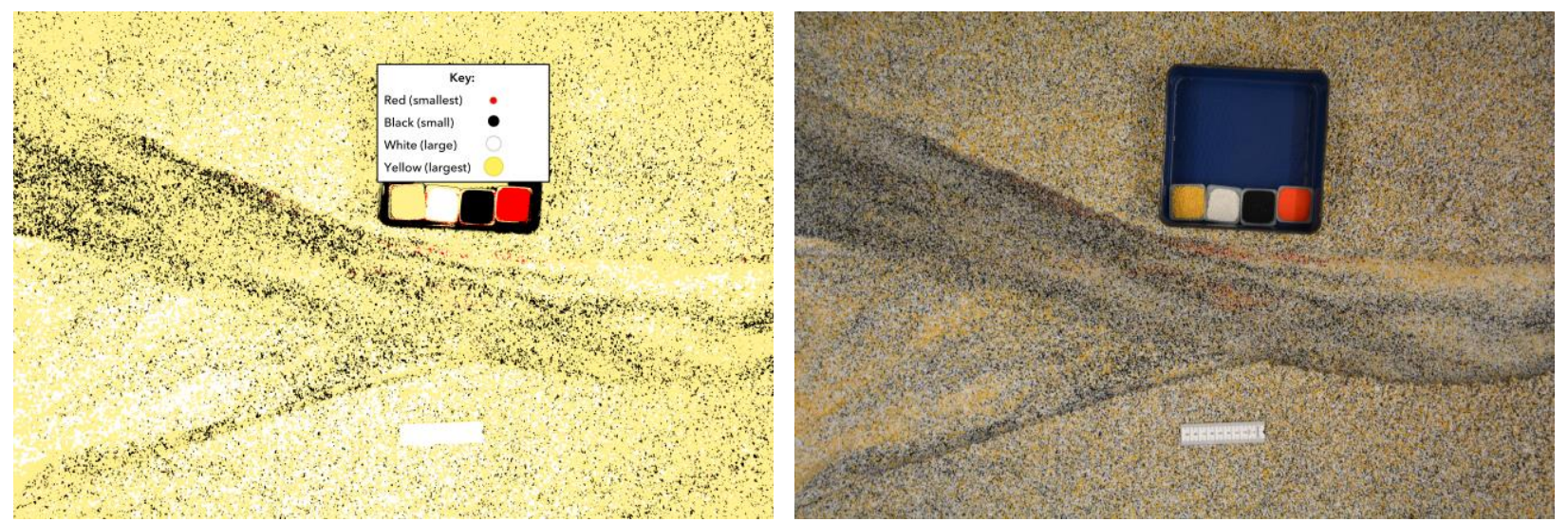

Figure 6 - Classified image of color sediment (left) and original color image (right). The classified image is used to calculate the proportion of area covered by each size class (color) of sediment. Reference samples of each color and a millimeter scale are included in each image.

At its core, the Weka Segmentation is a supervised classification routine where the user selects areas of known composition tells the computer what they are e.g. "forest", "cornfield", or in our case "red sediment". Then the computer uses algorithms to sort each pixel into one of the userselected categories (e.g. red, white, black, yellow). Though we have the capabilities of developing robust statistics on the quality of the classification, this is beyond the scope of this particular 
course, so we use a visual trial and error iteration to get most of the target pixels classified correctly.

\section{Course 2: GEOL 220 - Remote Sensing and GIS (12 students)}

Any introductory textbook on remote sensing will include a discussion of system parameters or resolutions (e.g. spatial, temporal, radiometric and spectral) as well as system operational characteristics (active or passive, scanning or framing). To reinforce these concepts and to understand the tradeoffs among them we use the Kinect and a digital camera as examples of remote sensing instruments. The Kinect, for example, is an active framing system. However, it is does not use time of flight to measure distance like conventional RADAR and LiDAR. For each instrument the students develop a spec sheet and compare them to the operations page of spacebased assets (for example see the HiRISE specs - www.uahirise.org/teknikos.php). Comparisons of the cameras from the Viking orbiters give students a new appreciation for how far technology has come. Students also take the system specs (e.g. CCD array size and focal length) from the DSLR and determine the spatial resolution of a pixel at a known distance. Then they are asked to evaluate how the field of view and resolution change as the focal length changes. They can verify their calculations by simply looking at the live field of view and importing the images into image processing/viewing software such as FIJI (Schindelin and others, 2012) or ArcGIS (ESRI, 2011). This exercise drives home the trade-off between spatial resolution and field of view.

Students are also engaged with important image processing techniques as they analyze the data taken from the Kinect and DSLR. The raw data has several artifacts such as infrared shadow and striping (for example Figure 5) which need to be removed through the application of various filters before analysis. Students rarely encounter such errors when browsing remotely sensed imagery because those images have already been corrected, so this gives them first hand experience with what happens behind the scenes. It is also an opportunity to explore the effects of different filters and to think about which one is best for vertically oriented artifacts. Students then need to calibrate the vertical and horizontal scales and classify the images using the Weka Segmentation procedure already described (e.g Figure 6). These are authentic image processing techniques that are made relevant because the students took their own data using devices.

These activities are metered out over several class periods and feedback is provided. Additionally we make frequent reference to these exercises as we discus the design and application of remote sensing platforms such as MODIS and HiRISE. As part of a final exam students are given a problem that asks them to determine a) if the Kinect can resolve the red sediment grains (mean diameter $=0.25 \mathrm{~mm}$ ) and $\mathrm{b}$ ) the optimal height from which to hang the digital camera to provide maximal coverage of the model flume while still being able to resolve the finest, red particles. Both of these questions require application of concepts learned earlier in the course and demonstrate mastery of the material.

\section{Learning Outcomes}

Due to our small sample sets $(n=24)$, and lack of a control group we rely on authentic assessment techniques to evaluate the effectiveness of our learning outcomes. Below each goal is discussed.

It is surprising considering the number of technologically savvy students, how few understand the fundamental constraints and uses of remotely sensed data. It is perhaps even more surprising that 
students in spatially dependent fields such as geology and environmental science are little better informed. Although one of the primary goals of this project was to introduce students in traditionally field-based disciplines to applications of remote sensing, this project far exceeded my expectations. The main benefit came from using common consumer electronic devices and casting them as remote sensing instruments. For example students were excited to learn how their digital cameras work and this motivated them to persevere through fairly complex problems - such as optimizing our remote sensing system and quantifying topographic changes in the model.

The comprised of the physical model and novel remote sensing system required students to engage directly with data collection, data reduction, and data analysis - all with goal of better understanding surficial processes. Although no quantitative data was collected to show improved quantitative reasoning, working through the problems and labs themselves was excellent practice. And students demonstrated ability to apply concepts learned both within and across courses. For example, students in the surficial processes class were asked to apply a new sediment transport law (aeolian rather than fluvial) to a new environment - Mars. Completing this problem requires that students understand the fundamental driving forces and how they differ in the two environments. By and large students did well on this problem. However, the most exciting gains were those by the students who took both courses.

The three students who took both courses benefitted the most because the each course used the technology in a different way and with different goals. In remote sensing, the main goal was learning the technology and the techniques, whereas in the surficial processes course the instrumentation was secondary and study of the processes was primary. Students who had remote sensing first were able to apply what they had learned and carry it over to the surface processes class. The most gratifying thing was that they also eagerly assisted other students with the analysis of terrestrial and model landforms. This 'accidental' peer teaching was very effective and generated additional interest in the remote sensing course. Likewise remote sensing students became interested in surficial processes and physical modeling, so much so that a special topics course on physical modeling was created and will be offered in the 2014-15 academic year.

\section{Future Work}

Though this work was successful, the image processing activities in the surficial processes class took much longer than anticipated. As a result we didn't have time to introduce a similar module on mass wasting. Rather that material was covered in the field and classroom. However, there is potential to reconfigure the class to run with two labs a week, which would allow enough time to expand the hill slope processes portion of the class and to incorporate modeling activities and remote sensing data. If time resources allow, the course will be offered as a double lab in 201516. I am also exploring with a colleague developing a version of the river modeling labs that would be appropriate for introductory geology. This will entail substantially simplifying the data processing so that students can focus on the processes demonstrated rather than image correction protocols.

\section{References}

American Association for the Advancement of Science (AAAS), 1989, Project 2061, Science for all Americans, 
AAAS Press, Washington, DC.

American Geophysical Union (AGU), 1997, Shaping the future of undergraduate earth science education: Innovation and change using an earth system approach, Washington, DC.

The Boyer Commission on Educating Undergraduates in the Research University, 1998, Reinventing Undergraduate Education: A Blueprint for America's Research Universities, Carnegie Foundation for the Advancement of Teaching, Princeton, NJ.

ESRI (Environmental Systems Resource Institute). 2011. ArcMap 10.1. ESRI, Redlands, California.

Hall, M., Frank, E., Holmes, G., Pfahringer, B., Reutemann, P., and Witten, I., 2009, The WEKA Data Mining Software: An Update, SIGKDD Explorations 11(1).

Leopold, L.B., and Wolman, M. G., 1960, River Meanders, Bulletin of the Geological Society of America, 71:769794.

Mankoff, K. D., and Russo, T. A., 2012, The Kinect: A low-cost, high-resolution, short-range 3D camera, Earth Surface Processes and Landforms Online. 11p.

National Research Council (NRC), 1997, Science Teaching Reconsidered: A Handbook Committee on Undergraduate Science Education, National Academy Press, Washington, DC.

Pain, C.F., and C.D. Ollier, 1995, Inversion of relief - a component of landscape evolution,. Geomorphology, 12(2):151-165.

Richeson, D., (http://divisbyzero.files.wordpress.com/2009/11/meander.png), accessed 7/29/2014.

Schindelin, J., Arganda-Carreras, I., Frise, E., Kaynig, V., Longair, M., Pietzsch, T., Preibisch, S., Rueden, C., Saalfeld, S., Schmid, B., Tinevez, J., White, D., Hartenstein, V., Eliceiri, K., Tomancak P., and Cardona, A., 2012, Fiji: an open-source platform for biological-image analysis, Nature Methods 9(7): 676-682.

Williams, G. P., 1986, River Meanders and Channel Size, Journal of Hydrology, 88:147-164. 